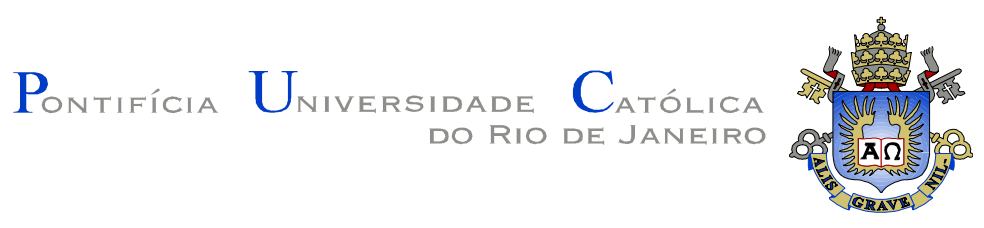

Mariana Souza Pires de Amorim

\title{
O Novo Brutalismo de Alison e Peter Smithson Em Busca da Ordem Espontânea da Vida
}

\section{Dissertação de Mestrado}

Dissertação apresentada como requisito parcial para obtenção do grau de Mestre pelo Programa de PósGraduação em História Social da Cultura do Departamento de História do Centro de Ciências Sociais da PUC-Rio.

Orientador: Prof. João Masao Kamita

Rio de Janeiro, Novembro de 2008 


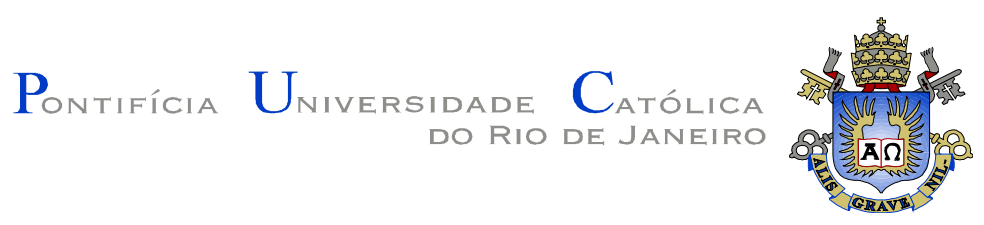

Mariana Souza Pires de Amorim

\begin{abstract}
O Novo Brutalismo de Alison e Peter Smithson:
Em Busca de Ordem Espontânea da Vida
\end{abstract}

Dissertação apresentada como requisito parcial para obtenção do grau de Mestre pelo Programa de Pós-Graduação em História Social da Cultura do Departamento de História do Centro de Ciências Sociais da PUC-Rio. Aprovada pela Comissão Examinadora abaixo assinada.

Prof. João Masao Kamita

Orientador

Departamento de História

PUC-Rio

Prof ${ }^{\mathrm{a}}$ Maria Cristina Nascentes Cabral

Faculdade de Arquitetura e Urbanismo UFRJ

Prof. Otávio Leonídio Ribeiro Departamento de Artes e Design

PUC-Rio

Profo Nizar Messari

Vice-Decano de Pós-Graduação do Centro de Ciências Sociais

PUC-Rio

Rio de Janeiro, 19 de novembro de 2008. 
Todos os direitos reservados. É proibida a reprodução total ou parcial do trabalho sem autorização da universidade, da autora e do orientador.

\section{Mariana Souza Pires de Amorim}

Graduou-se em Arquitetura e Urbanismo na UFRJ em 2004. Cursou Especialização em História da Arte e Arquitetura no Brasil na PUC - Rio em 2006.

Ficha Catalográfica

Amorim, Mariana Souza Pires de

O novo brutalismo de Alison e Peter Smithson em busca da ordem espontânea da vida / Mariana Souza Pires de Amorim ; orientador: João Masao Kamita. - 2008.

154 f. : il. ; $30 \mathrm{~cm}$

Dissertação (Mestrado em História)Pontifícia Universidade Católica do Rio de Janeiro, Rio de Janeiro, 2008.

Inclui bibliografia

1. História - Teses. 2. História social da cultura. 3. Novo brutalismo. 4. Arquitetura moderna. 5. Alison Smithson. 6. Peter Smithson. I. Kamita, João Masao. II. Pontifícia Universidade Católica do Rio de Janeiro. Departamento de História. III. Título.

CDD: 900 


\section{Agradecimentos}

Ao meu orientador Professor João Masao Kamita pela compreensão e parceria para a realização deste trabalho.

À Capes e à PUC-Rio, pelos auxílios concedidos, sem os quais este trabalho não poderia ter sido realizado.

A minha mãe pela contribuição com as traduções além do apoio, carinho e estimulo.

A minha tia Eduarda e meu tio Alfredo pelo interesse, compreensão e apoio afetivo e material.

A minha avó Maria José pelas orações e oa meu irmão Pedro pela contribuição com as imagens.

Aos meus amigos Rafael e Myriam por todo apoio e paciência. A minha mais nova amiga Mara por ter me acolhido em sua casa em Londres e por ter me apresentado a cidade.

Aos professores que participaram da banca examinadora.

A professora Ana Luisa Nobre pela contribuição de material fundamental para a construção deste trabalho.

A todos os professores e funcionários do Departamento, em especial a Edna Timbó pela dedicação e ajuda.

A biblioteca da PUC-Rio pela presteza com que responderam aos meus pedidos. 


\section{Resumo}

Amorim, Mariana Souza Pires de; Kamita, João Masao. O Novo Brutalismo de Alison e Peter Smithson: Em Busca da Ordem Espontânea da Vida. Rio de Janeiro, 2008. 154p. Dissertação de Mestrado - Departamento de História, Pontifícia Universidade Católica do Rio de Janeiro.

Alison e Peter Smithson foram os precursores do movimento arquitetônico chamado de Novo Brutalismo que surgiu na Inglaterra no ano de 1953. Interessados pelo Movimento Moderno em Arquitetura participaram dos últimos Congressos do CIAM, do qual se desligaram para integrar o grupo Team 10. Começaram suas carreiras no período do pós-guerra quando além de ser necessário reconstruir parte da Europa bombardeada, foi preciso adequar as cidades para o uso dos veículos motorizados que começavam a invadi-las. Diante de tantas mudanças na vida das comunidades, sua grande preocupação foi a preservação da identidade destas comunidades, respeitando seus hábitos sociais e culturais. Este respeito pela vida, tradição e valores levou-os a propostas absolutamente revolucionárias para a época: Projeto Hunstanton School; Projeto Golden Lane; Projeto Universidade de Sheffield. Participaram do movimento artístico britânico "Independent Group" no qual fizeram as exposições "Parallel Of Life and Art" e "This is Tomorrow". Podemos definir seu trabalho e suas ideologias com suas próprias palavras: "a arquitetura surge como resultado direto de um sentimento de vida".

\section{Palavras - chave}

Novo Brutalismo; Arquitetura Moderna; Alison Smithson; Peter Smithson. 


\section{Abstract}

Amorim, Mariana Souza Pires de; Kamita, João Masao. The New Brutalism of Alison and Peter Smithson: The Search of Spontaneous Order of Life. Rio de Janeiro, 2008. 154p. MSc Dissertation Departamento de História, Pontifícia Universidade Católica do Rio de Janeiro.

Alison e Peter Smithson were the predecessors of the Architectural Movement called New Brutalism that happened to appear in England in 1953. They were involved with the Modern Architectural Movement and they took part in the last CIAM Congresses, but broke up with it and organized themselves into a new group - Team 10. They began their careers in the post-war period when besides being necessary to reconstruct part of Europe which had been devastated by bombs, it was necessary to adapt the cities to the use of motor vehicles which were beginning to invade them. In face of so many changes in the communities lives they were very concerned in sustaining the identity of these communities, respecting their culture and social values. This respect for life, tradition and values led them to propose absolutely revolutionary for their time: Hunstanton School; Golden Lane Project; University of Sheffield Project. They took part in the British movement "Independent Group" within which they made the exhibitions "Parallel of Life and Art" and "This is Tomorrow". We can define their work and their ideas with their own words: "we see architecture as the direct result of a way of life."

\section{Keywords}

New Brutalism; Modern Architecture; Alison Smithson; Peter Smithson. 


\section{Sumário}

$\begin{array}{ll}\text { Introdução } & 11\end{array}$

1. A Situação Britânica no Pós-Guerra 15

1.1. O Plano para Grande Londres 15

1.2. New Town 17

1.3. Architect's Department of the London County Council - LCC 21

1.4. Novos Palladinos ou Novos Acadêmicos 24

1.5. Antropologia, Sociologia e Etnologia na Sociedade Britânica 27

2. Atitude Formalista $\quad 34$

2.1. Escola Secundária Hunstanton 34

3. Padrões de Associação e Identidade 44

3.1. Urban Re-Identification - 9 CIAM - 1953

3.2. An Urban Project 48

3.3. Urban Structuring 52

3.4. Golden Lane 63

3.5. Sheffield University $\quad 72$

3.6. Casa do Futuro 80

4. Novo Brutalismo nas Artes 86

4.1. Independent Group 86

4.2. Parallel of Life and Art 91

4.3. This Is Tomorrow 98

5. Manifestos Brutalista 104

5.1. Manifesto Brutalista 104

5.2. Statement of Principles 106

5.3. Novo Brutalismo 108

6. O Brutalismo e o Team $10 \quad 110$

6.1. CIAM 9 e antecedentes 110

6.2. Preparação para CIAM $10 \quad 112$

6.2.1. Manifesto Doorn 113 
6.2.2. Urban Re-Identification 115

6.3. CIAM 10 e Team $10 \quad 118$

6.3.1. Cluster City 120

6.3.2. Otterlo Statement 121

$\begin{array}{ll}\text { Conclusão } & 123\end{array}$

$\begin{array}{ll}\text { Referências Bibliográficas } & 124\end{array}$

$\begin{array}{ll}\text { Apêndice } & 127\end{array}$

An Urban Project - artigo original 128

An Urban Project - tradução livre 135

Urban Re-Identification - artigo original 138

Urban Re-Identification - tradução livre $\quad 142$

$\begin{array}{lr}\text { Cluster City - artigo original } & 145\end{array}$

Cluster City - tradução livre $\quad 149$

Manifesto Doorn 153

Otterlo Statement - artigo original 154 


\section{Lista de figuras}

Fig. 01 - Conjunto de casas de uma New Town. 20

Fig. 02 - Alton Estate em Roehampton. 24

Fig. 03 - Sede do Pionner Health Centre. 28

Fig. 04 - Interior do Centro: cafeteria e piscina. 30

Fig. 05 - Sede em um dia de festa. 31

Fig. 06 - Croqui do Centro Peckham. 32

Fig. 07 - Fotografia do conjunto de Hunstanton. 36

Fig. 08 - Desenho do conjunto de Hunstanton. 36

Fig. 09 - Hall e acessos ao primeiro pavimento de Hunstanton. $\quad 37$

Fig. 10 - Interior do hall de Hunstanton. 38

Fig. 11 - Pátio interno de Hunstanton. 39

Fig. 12 - Pias no lavatório de Hunstanton 40

Fig. 13 - Planta do pavimento térreo de Hunstanton.

Fig. 14 - Planta do primeiro pavimento de Hunstanton. 43

Fig. 15 - Planta do ginásio de Hunstanton: térreo e $1^{\circ}$ pavimento.

Fig. 16 - Painel Urban Re-Identification preparado pelos Smithson para o 9 Congresso do CIAM em Aix-en-Provence em 1953.

Fig. 17 - Bethnal Green 1956 - Foto de Nigel Henderson. $\quad 45$

Fig. 18 - Parte do painel de Urban Re-Identification. 46

Fig.19 - Imagens do livro Urban Structuring

- fotos de Nigel Henderson. 52

Fig. 20 - Planta de situação de Golden Lane. 65

Fig. 21 - Planta do nível abaixo ou acima da street deck - unidade básica dos pais. 66

Fig. 22 - Planta do nível da street deck. $\quad 67$

Fig. 23 - Housing in Spangen, Rotterdam, Michiel Brinckman, 1920. 
Fig. 24 - Montagem (desenho e fotos) da street deck de Golden Lane.

Fig. 25 - Desenho da elevação leste de Golden Lane.

Fig. 26 - Desenho da elevação norte de Golden Lane. $\quad 71$

Fig. 27 - Situação de Sheffield: blocos existentes em preto. 73

Fig. 28 - Novos volumes de Sheffield em preto. 73

Fig. 29 - Sheffield: declive do terreno.

Fig. 30 - Perspectiva dos novos blocos de Sheffield. 76

Fig. 31 - Elevação do bloco de Artes e Administração de Sheffield.

Fig. 32 - Perspectiva do novo bloco e das ruas aéreas de Sheffield. 78

Fig. 33 - Perspectiva da Casa do Futuro. $\quad 80$

Fig. 34 - Planta da Casa do Futuro. $\quad 80$

Fig. 35 - Cômodo de vestir com os nichos. 82

Fig. 36 - Cadilac "ícone pop".

Fig. 37 - Foto da maquete da Casa do Futuro. 85

Fig. 38 - Foto da exibição Parallel of Life and Art. 92

Fig. 39 - Foto da exibição Parallel of Life and Art. 93

Fig. 40 - Capa do catálogo da exibição

Parallel of Life and Art. $\quad 94$

Fig. 41 - Perspectiva do projeto para

Patio and Pavilion. $\quad 99$

Fig. 42 - Fotografia da exibição Patio and Pavilion 100

Fig. 43 - Fotografia da exibição Patio and Pavilion. 101

Fig. 44 - Imagem do catálogo This is Tomorow. 102

Fig. 45 - Foto da exibição Patio and Pavilion. 103

Fig. 46 - Imagem do catálogo This is Tomorow com os componentes do Grupo 6. 